\title{
Amplitude noise suppression in high-repetition-rate pulse train generation from a frequency-modulated Er-Yb laser
}

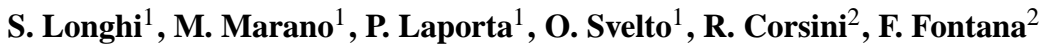 \\ ${ }^{1}$ INFM, Dipartimento di Fisica del Politecnico di Milano and CEQSE-CNR, P. zza L. da Vinci 32, 20133 Milano, Italy \\ (Fax: +39-02/2399-6126, E-mail: longhi@ fisi.polimi.it) \\ ${ }^{2}$ Pirelli Cavi \& Sistemi S.p.A., Viale Sarca 222, 20126 Milano, Italy
}

Received: 28 July 1999/Revised version: 13 September 1999/Published online: 20 October 1999

\begin{abstract}
A detailed experimental analysis of the amplitude noise in high-repetition-rate picosecond pulse trains generated by spectral filtering of a frequency-modulated ErYb:glass laser is reported. Two distinct sources of noise are identified, and stabilization techniques for noise suppression are proposed and experimentally demonstrated. Intensity noise suppression of $\sim 20 \mathrm{~dB}$, corresponding to less than $2 \%$ residual amplitude fluctuations of the pulse train, has been achieved at repetition rates of $2.5 \mathrm{GHz}$ and $5 \mathrm{GHz}$ with pulse durations of $\sim 50 \mathrm{ps}$.
\end{abstract}

PACS: 42.60.Fc; 42.60.Rn

The generation of high-repetition-rate picosecond pulse trains at $1.5 \mu \mathrm{m}$ is currently a topic of great importance in solitonbased optical transmission systems [1]. Active mode-locking methods, based on intracavity amplitude or frequency modulation, are attractive for their capability of generating symmetric and chirp-free pulses suitable for soliton transmissions, but they usually fail in producing pulse trains with long-term stability owing to their extreme sensitivity to cavity length variations, requiring some nontrivial form of active stabilization $[2,3]$. We recently proposed $[4,5]$ a novel nonresonant technique to generate high-repetition-rate pulse trains with controllable pulse duration, attractive for its high tolerance to cavity length fluctuations with respect to conventional active mode-locking methods. The technique is based on suitable spectral filtering [6] of the optical field emitted by an intracavity frequency-modulated (FM) laser [7], in which the modulation frequency $f_{\mathrm{m}}$ is close but not equal to the cavity axial mode separation $f_{\mathrm{c}}$ or, in the so-called harmonic regime, to one of its harmonics. The feasibility of this technique to generate transform-limited pulse trains at a repetition rate equal to or twice the modulation frequency was previously demonstrated by using a compact Er-Yb:glass laser FM-operated in a third-harmonic configuration $[4,5]$. Although the nonresonant modulation technique makes the pulse trains largely insensitive to cavity length variations, the asynchronous regime of operation of the laser deserves special attention from the point of view of possible dynamical instabilities [8] that could be detrimental in soliton transmissions.

In this article we describe the experimental characterization of the amplitude noise detected in the pulse trains generated by an FM-operated Er-Yb:glass laser at $1533 \mathrm{~nm}$ and the stabilization techniques used for the noise suppression, and we report on the results achieved. The experimental layout for the nonresonant generation of the pulse trains is similar to that previously reported [4] and consists of an intracavity phase modulated Er-Yb:glass laser and an external spectral filtering stage. The laser cavity consists of a compact twomirror hemispherical resonator that is about $47 \mathrm{~mm}$ long, with a 1-mm-thick Er-Yb:glass disk, end-pumped at $980 \mathrm{~nm}$ by an InGaAs laser diode, which forms one end of the resonator [9]. A resonant $\mathrm{LiNbO}_{3}$ phase modulator placed inside the cavity is used to operate the laser in the fundamental-order FM regime at a modulation frequency of $2.5 \mathrm{GHz}$. An uncoated $120-\mu \mathrm{m}$-thick Polarcor etalon is also inserted in the cavity with the double aim of ensuring the field polarization along the $z$ axis of the $\mathrm{LiNbO}_{3}$ crystal and of controlling the carrier frequency of the laser emission. The laser output is coupled through a microscope objective into a single-mode fiber and sent, through a three-port optical circulator, to a 1-cm-long fibre Bragg grating, designed to operate in the reflection configuration as a narrow bandpass filter. The mean optical power at the output, for a fiber coupling efficiency of $40 \%$, is $\sim 0.5 \mathrm{~mW}$, corresponding to $\sim 10 \%-15 \%$ of the overall fiber-coupled power of the FM signal. The pulse duration can be easily tuned, by fine adjustement of the detuning frequency, from $90 \mathrm{ps}$ down to $40 \mathrm{ps}$. Time-bandwidth products, as derived from spectral and autocorrelation measurements, indicate that the pulses are close to the Fourier transform limit.

In order to assess the suitability of the laser source for soliton transmissions, a detailed analysis of short- and longterm stability of the pulse trains has been performed. Two noise sources, corresponding to distinct phenomena, have been found to affect the pulse train envelope. These include amplitude fluctuations due to relaxation oscillations, typically in the range of tens of $\mathrm{kHz}$, and amplitude oscillations at the detuning frequency $d f=\left|f_{\mathrm{m}}-f_{\mathrm{c}}\right|$ and harmonics, typ- 
ically in the range of a few MHz. The former source of noise is commonly found in other mode-locked erbium laser systems (see, for instance, [10]), and, in our typical experimental conditions, it is not appreciably influenced by the asynchronous mode of operation of the laser. Relaxation oscillation noise causes amplitude fluctuations $(\sim 10 \%-20 \%$, peak-to-peak value) of the pulse train envelope on a time scale of $\sim 10-30 \mu \mathrm{s}$, mainly ascribable to fluctuations of cavity losses and amplitude noise of the pump laser. The relative intensity noise (RIN) spectrum of the laser output exhibits a characteristic peak around the relaxation frequency, which can vary between 35 and $90 \mathrm{kHz}$, depending on the pump power level. A typical behaviour of the RIN spectrum is shown in Fig. 1a (dashed line), and the corresponding signal in the time-domain is reported in Fig. 1b (dashed line). We measured a typical $\sim 10 \%$ peak-to-peak amplitude modulation, compared to the DC value, which is superimposed on the pulse train envelope.

Amplitude oscillations at the detuning frequency (typically $2-4 \mathrm{MHz}$ ), with modulation depths of $\sim 15 \%-25 \%$ (peak-to-peak value), were also observed on the pulse train envelope. Figure 2a (dashed line) shows a typical RF noise spectrum of the pulse train in the $\mathrm{MHz}$ range, detected by a low-noise photodiode (New Focus Model 1811, bandwidth $125 \mathrm{MHz}$ ), which exhibits a high peak at $2.9 \mathrm{MHz}$ and a lower second-harmonic peak at 5.8 MHz. The detuning frequency was $2.9 \mathrm{MHz}$, corresponding to an oscillating bandwidth of $130 \mathrm{GHz}$ and to a pulse duration of $\sim 50 \mathrm{ps}$. The corresponding time-domain signal is reported in Fig. 2b (dashed line). This source of noise has a much more involved phys-
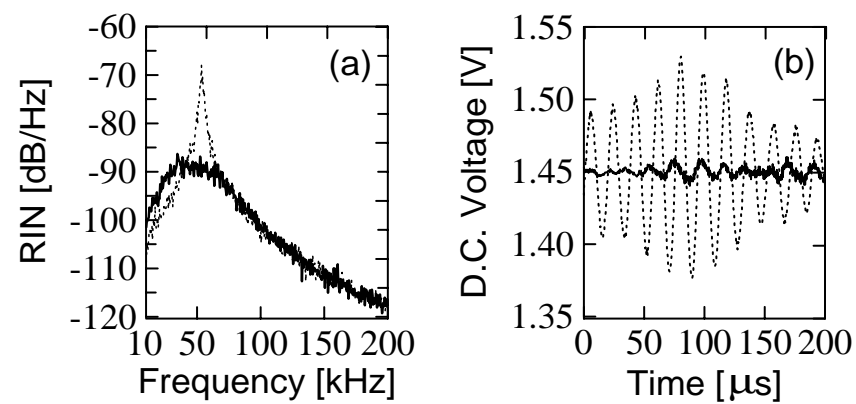

Fig. 1a,b. Relative intensity noise (RIN) spectrum at around the relaxation oscillation frequency (a), and corresponding time-domain signal (b) of the pulse train envelope with the feedback circuit on (solid line) and off (dashed line)
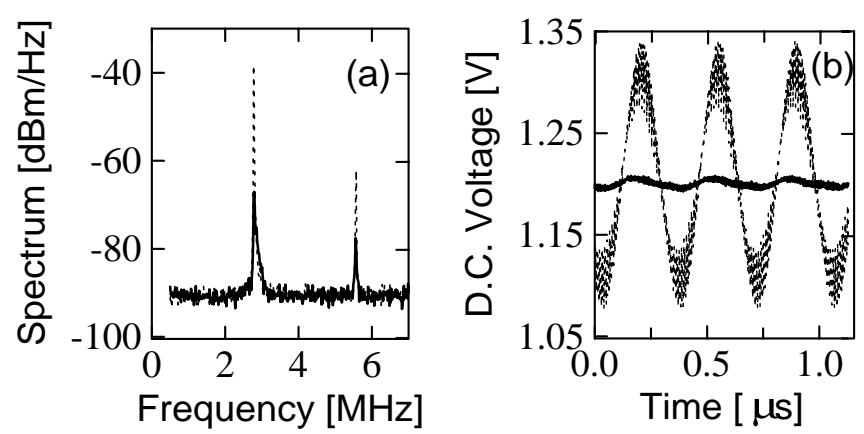

Fig. 2a,b. Power spectrum (a), and time-domain behaviour of the pulse train envelope in the $\mu$ s time scale (b), showing amplitude noise due to frequency detuning with the feedback circuit on (solid line) and off (dashed line) ical origin related to the asynchronous mode of operation of the phase modulator [11]. It is probably ascribable to at least two different mechanisms: mode-hopping among longitudinal modes of the frequency-modulated cavity [7], induced by environmental perturbations, on the one hand, and strong excitability of the laser dynamics at the detuning frequency and harmonics, leading to enhanced amplification of the noise level at these resonances, on the other hand. The excitability of the laser dynamics at the detuning frequency and harmonics is basically a resonance phenomenon related to the fact that distinct FM cavity modes have spectral components separated by the detuning frequency and multiplies [7]. In addition, especially at low values of the detuning, the FM modes become highly distorted and their non-orthogonal character is responsible for an enhanced sensitivity of the system to noise. In fact, it can be shown that an intracavity frequency-modulated laser operated close to threshold can sustain a large excess of field fluctuations as a result of the non-orthogonality of laser modes. In particular, the excess of noise variance can approach values as high as $10^{8}$ at a point corresponding to the transition region between FM oscillation and FM mode-locking, leading to a huge transient amplification of perturbations [11]. Evidence of noisy behaviour of FM-operated lasers is indeed reported in the literature [8]. Experimentally, we have found in our case that the noise level, as expected, is largely dependent on detuning, increasing as the detuning parameter is decreased until marked noisy behaviour is observed corresponding to the transition region between FM oscillation and FM mode-locking. However, typical detuning values of interest for pulse train generation $(d f \gtrsim 2.5 \mathrm{MHz})$ are rather far away from this highly unstable regime, which occurs below $d f \sim 0.6 \mathrm{MHz}$, so that it is not of concern in the present study.

In order to suppress relaxation oscillation and frequency detuning noise, active stabilization based on a double control loop was carried out. As far as relaxation oscillation noise is concerned, a feedback circuit acting on the pump power was designed on the basis of the measured transfer function (both in amplitude and in phase) between pump power and laser output $[12,13]$. Figure 3 shows a schematic diagram of the control feedback loop: a small fraction $(\sim 5 \%)$ of the output power is sent to a low-noise photodiode, the output of which is connected to a bandpass filter, F1, with adjustable central frequency $(\sim 30 \mathrm{kHz}$ bandwidth at $-3 \mathrm{~dB})$ that provides high gain at the relaxation oscillation frequency. The electrical signal is then sent to the current driver of the pump laser diode; the resulting open loop gain is $\sim 20 \mathrm{~dB}$ at the relaxation peak. Figure 1a shows the measured low-frequency noise spectrum of the laser with the feedback circuit turned on (solid line); Fig. $1 \mathrm{~b}$ also depicts the corresponding time-domain signal. The peak suppression is $\sim 20 \mathrm{~dB}$ and the residual RIN is below $\sim-88 \mathrm{~dB} / \mathrm{Hz}$; notice that, with the control circuit on, the total peak-to-peak amplitude fluctuation is less than $1.5 \%$.

In order to suppress the amplitude noise due to frequency detuning, we exploited the fact that in the asynchronous mode of operation the laser is easily excitable at the detuning frequency and harmonics. In particular, we found that the laser output is strongly sensitive to weak modulations, at the detuning frequency, of the RF power supplied to the intracavity phase modulator. We therefore designed a second feedback circuit acting on the RF signal and composed by an adjustable 


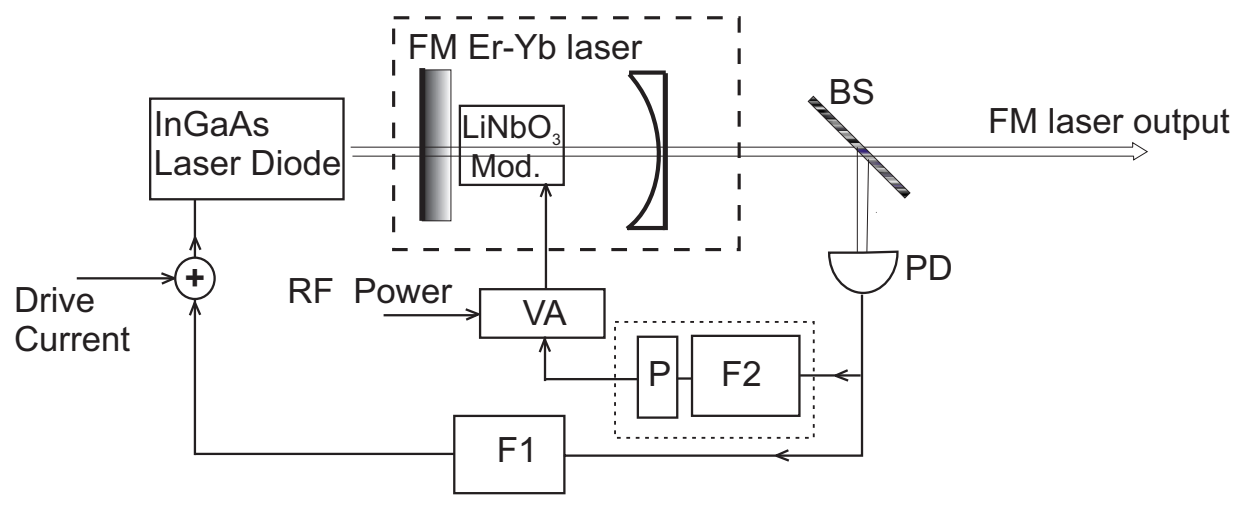

Fig. 3. Schematic diagram of the control feedback loops for amplitude noise suppression. BS, beam splitter; PD, lownoise photodetector; F1 and F2, bandpass filters at relaxation oscillation and detuning frequencies, respectively; $\mathrm{P}$, phase control; VA, variable attenuator

bandpass filter, F2, centered at the detuning frequency, followed by an additional phase control to achieve loop stability. As can be seen from Fig. 3, the electrical signal at the output of the low-noise photodiode is sent to the feedback circuit and then to the low-frequency control input of a GaAs MMIC variable attenuator acting on the RF power level which drives the phase modulator. The solid line in Fig. 2a shows a typical behaviour of the RF noise spectrum in the $\mathrm{MHz}$ range when the suppression circuit is turned on, indicating that a reduction of amplitude noise of more than $20 \mathrm{~dB}$ is obtained. In Fig. 2b (solid line) the corresponding time-domain signal is also reported. A residual peak-to-peak amplitude oscillation of the pulse train envelope less than $1.5 \%$ was achieved with the control feedback loop turned on. We note that this feedback control does not require any additional optical element inside or outside the resonator in order to stabilize the power level. The overall long-term stability of the pulse train envelope with the two feedback controls turned on is shown in Fig. 4, and compared with that with no feedback. In closedloop operation, no appreciable amplitude fluctuations were observed for more than one hour without particular care to reduce mechanical and acoustical noise being taken. It is worth pointing out that, for both the control circuits we realized, only low-frequency analogue electronic components are used, and neither complicated stabilization techniques nor computers are needed.

In order to assess the applicability of the feedback control system at higher repetition frequency, the proposed stabilization method has also been exploited to stabilize a dualwavelength pulse train generated using a different spectral filtering scheme [5], which enables doubling of the pulse repetition rate. While $2.5 \mathrm{GHz}$ or $5 \mathrm{GHz}$ appears to be an upper limit for operation in the fundamental harmonic regime, it

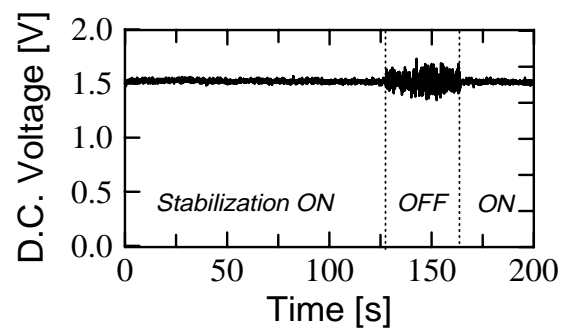

Fig. 4. Long-term behaviour of the pulse train envelope as measured on a digital oscilloscope with control feedback loops turned on, off and on again should be noticed that the achievement of higher repetition rates is still possible [14], but the laser would have to be operated in a harmonic regime. A detailed experimental analysis of the noise characteristics for a $2.5-\mathrm{GHz}$ pulse train generated in a laser configuration operated in the third-order harmonic [4] indicated that, both with and without operated feedback loops, the noise levels at relaxation oscillation and detuning frequencies are not influenced by the harmonic operation regime of the laser and the results discussed above are still valid. In this case, however, a third source of noise, related to supermode competition, is sometimes observed. Although in our case the relatively low harmonic order (thirdorder) enables us to reduce the occurrence of mode-hopping by careful isolation of the laser from environmental disturbances, supermode beating might remain a serious problem when the laser is operated at larger harmonic orders, and effective suppression of such instability would require, for instance, an intracavity spectral selective element. The same problems, however, arise with waveguide lasers [15] and fibre lasers [16]. Fibre lasers, in particular, in addition to being susceptibe to mechanical vibrations and temperature variations affecting the cavity length, also operate at very high (from hundredths to thousandths) harmonics of the cavity mode spacing, and therefore they need effective suppression of supermode noise. Although different techniques have been proposed and successfully demonstrated to this aim, they turn out to be generally rather complex.

In conclusion, a detailed investigation of the noise properties of pulse trains generated by spectral filtering of an FM-operated Er-Yb:glass laser has been presented, and stabilization techniques for noise suppression have been proposed and experimentally demonstrated. The high stability of the pulse trains on short and long time scales, together with the absence of chirp and the tunability of pulse duration, makes this laser source of interest for its potential use in solitonbased transmission systems.

Acknowledgements. This research was partially supported by Italian National Research Council (CNR) under the "Progetto Finalizzato MADESS II".

\section{References}

1. Special issue on ultrafast optical pulse technologies and their applications, IEICE Trans. Electron. E 81-C, 93 (1998)

2. T. Harvey, L.F. Mollenauer: Opt. Lett. 18, 107 (1993)

3. M. Nakazawa, E. Yoshida, Y. Kimura: Electron. Lett. 30, 1603 (1994) 
4. S. Longhi, S. Taccheo, P. Laporta: Opt. Lett. 22, 1642 (1997)

5. S. Longhi, G. Sorbello, S. Taccheo, P. Laporta: Opt. Lett. 23, 1547 (1998)

6. P.V. Mamyshev: Opt. Lett. 19, 2074 (1994)

7. S.E. Harris, O.P.Mc. Duff: IEEE J. Quantum. Electron. QE-1, 245 (1965)

8. A. Schremer, T. Fujita, C.F. Lin, C.L. Tang: Appl. Phys. Lett. 52, 263 (1988)

9. S. Longhi, S. Taccheo, P. Laporta, C. Svelto: Electron. Lett. 31, 368 (1995)

10. H. Takara, S. Kawaniski, M. Saruwatari: Electron. Lett. 31, 292 (1995)
11. S. Longhi, P. Laporta: Excess noise in intracavity laser frequency modulation (unpublished)

12. T.J. Kane: IEEE Phot. Technol. Lett. 2, 244 (1990)

13. S. Taccheo, P. Laporta, O. Svelto, G. De Geronimo: Opt. Lett. 21, 1747 (1996)

14. S. Longhi, G. Sorbello, S. Taccheo, P. Laporta, R. Corsini, F. Fontana: Conference on Lasers and Electro-Optics '99, paper CThA7, (Baltimora, MA 1999) p. 352

15. H. Suche, A. Greiner, W. Qiu, R. Wessel, W. Sohler: IEEE J. Quantum. Electron. QE-33, 1642 (1997)

16. X. Shan, D. Cleland, A. Ellis: Electron. Lett. 28, 182 (1992) 